\section{What to do when the robots come? Non-formal education in jobs affected by automation}

Non-formal education and job automation risk

\author{
Sierdjan Koster
}

Department of Economic Geography, Faculty of Spatial Sciences, University of Groningen, Groningen, The Netherlands, and

Claudia Brunori

European University Institute, Fiesole, Italy
1397

Received 30 June 2020

Revised 1 December 2020 18 March 2021

Accepted 22 March 2021

\begin{abstract}
Purpose - Ongoing automation processes may render a fair share of the existing jobs redundant or change their nature. This begs the question to what extent employees affected invest in training in order to strengthen their labour market position in times of uncertainty. Given the different national labour market regimes and institutions, there may be an important geographical dimension to the opportunities to cope with the challenges set by automation. The purpose of this study is to address both issues.

Design/methodology/approach - Using data from the 2016 European labour Force Survey, the authors estimate with logit and multi-level regression analyses how the automation risk of a worker's job is associated with the propensity of following non-formal education/training. The authors allow this relationship to vary across European countries.

Findings - The results show that employees in jobs vulnerable to automation invest relatively little in training. Also, there are significant differences across Europe in both the provision of training in general and the effect of automation on training provision.

Originality/value - While there is quite a lot of research on the structural labour market effects of automation, relatively little is known about the actions that employees take to deal with the uncertainty they are faced with. This article aims to contribute to our understanding of such mechanisms underlying the structural macro-level labour-market dynamics.
\end{abstract}

Keywords Training, Labour market, Automation

Paper type Research paper

\section{Introduction: automation and training}

Automation, fuelled by developments in artificial intelligence, adaptive automated learning and robotics, is a prime driver of current labour market dynamics. It leads to the loss and restructuring of current jobs, although the exact magnitude of the effect is heavily debated. At the one end, Brynjolfsson and McAfee (2014) and Frey and Osborne (2017) predict major job losses to automation. Frey and Osborne (2017) see up to $47 \%$ of US jobs as vulnerable to automation. At the other end, OECD studies by Arntz et al. $(2016,2017)$ and Nedelkoska and Quintini (2018) predict a more modest $9 \%-14 \%$ of all jobs in OECD countries to be at risk of becoming redundant in the face of automation.

(C) Sierdjan Koster and Claudia Brunori. Published by Emerald Publishing Limited. This article is published under the Creative Commons Attribution (CC BY 4.0) licence. Anyone may reproduce, distribute, translate and create derivative works of this article (for both commercial and non-commercial purposes), subject to full attribution to the original publication and authors. The full terms of this licence may be seen at: http://creativecommons.org/licences/by/4.0/legalcode

This study is based on data from Eurostat, European Labour Force Survey, 2016. The responsibility for all conclusions drawn from the data lies entirely with the authors.

Many thanks to Lianne Hans and Luuk Bos for their contributions to the data preparation and initial empirical analyses. 
IJM

42,8

The studies underlying these predictions take the technological frontier as their starting point; that is they explore the potential labour market effects of automation by intersecting the features of the technology and the tasks performed in jobs. The adoption of innovation with automation as an example, however, is importantly mediated by the potential of countries, sectors, organizations and workers to implement automation processes. With this, the urgency for workers to respond to the potential effects of automation will differ across contexts. At the level of the organization, for example, the implementation of new technologies and R\&D are influenced by the current set of skills available to the organization and also the organizational structure itself (Cohen and Levinthal, 1990; Piva and Vivarelli, 2009; Jiménez-Jiménez and SanzValle, 2005; Bresnahan et al., 2002). Similarly, at the sectoral level, it has been argued that the adoption of new technologies and the role of $R \& D$ is dependent on both the production function of sectors and historically grown common practices regarding the appropriation and use of new technologies (Capone et al., 2019; Malerba et al., 2016). In addition to the adaption of automation, also the employment effect of automation depends on how and to what extent it is implemented, both at the labour market level (Acemoglu and Restrepo, 2018, 2019) and at the firm level (see Calvino and Virgillito, 2018 for a review). Empirically, the net employment impact of innovations remains unclear (Dosi and Mohnen, 2019) with product innovation being suggested to lead to labour polarization without a clear effect on employment growth (Hou et al., 2019). Process innovation involves labour shedding more prominently (Pellegrino et al., 2019), even though this effect can be compensated by employment growth following the increased competitiveness of the firm or industry (Dosi and Mohnen, 2019). As an illustration, Graetz and Michaels (2018) show that the implementation of robots increases firm productivity and with that creates new jobs, although not for low-skilled workers. Summarizing the argument, the actual labour market impact of innovation, and automation specifically, is then a complex interplay of the features of the technological change, the organizational and sectoral ability to implement automation, and the generation of new jobs that are compatible with the new technology.

Even though the economic urgency to respond to automation may differ across contexts, it is clear that automation poses important challenges for current workers. Even the relatively modest predictions by Nedelkoska and Quintini (2018) suggest that 66 million workers in 32 OECD countries are active in soon-to-be redundant jobs. And, if automation leads to new jobs, they will have different features. Previous studies have highlighted how these asymmetric effect of automation processes between skilled and unskilled workers (and between people with a high and low socio-economic status) have led to increased income polarization (Acemoglu and Autor, 2011; Goos and Manning, 2007; Graetz and Michaels, 2018). However, the underlying mechanisms leading to this polarization remain largely unaddressed (Kerr et al., 2020).

In this article, we argue that one mechanism driving the asymmetric labour market effects of automation are differences in access to training - defined as non-formal education [1] between workers with higher and lower risks of losing their jobs to automation. The new tasks created by automation processes urge workers to update their skill set. Thus, investments in one's human capital, in the form of training attendance, are fundamental for workers to increase their employability, should they lose their jobs to automation or if they have to update their skill set in order to work with the new technology (Froehlich et al., 2014; Wittekind et al., 2010). Echoing this and underlining its relevance, ensuring the provision of non-formal education to all groups is a key goal in the European Employment Guidelines as drawn up by the European Committee (see Wilthagen and Tros, 2004).

To our knowledge, only one study (Nedelkoska and Quintini, 2018) has to date investigated the differences in training attendance between workers depending on their automation risk. Nedelkoska and Quintini (2018) find that workers who have higher automation risk are less likely to attend training than workers with lower risk of losing their jobs to automation. We expand on this finding by analysing country-level differences in the likelihood of training attendance for workers in general, and in the relative risk of training 
attendance between high- and low-automation risk workers. In addition, we investigate whether such country differences are due to national level labour market policies.

We look at country level differences because institutional differences have an important bearing on the provision of non-formal education (Acemoglu and Pischke, 1999). In addition, the exposure to automation differs across countries along the lines of distinct occupational profiles (see, for example, Arntz et al., 2016). Also, following the earlier discussion, adaption of automation technologies may run at different speeds given for example the current skill level of the work force. The economic urgency of ensuring employability of the affected workers likely varies across Europe as a result. As such, the study contributes to a better understanding of the geographically different impacts of automation processes on the labour market (Autor, 2019). To assess country level differences, we investigate our research question using data for $23 \mathrm{EU}$ countries from the 2016 round of the European Labour Force Survey (EU-LFS).

\section{The provision of non-formal education}

The relationship between automation and training pertains to a wider discussion regarding employability. Employability is the ability of people to identify and realize job and career opportunities (Fugate et al., 2004; Froehlich et al., 2014). Under the influence of globalization processes and technological change, labour markets increasingly ask for flexibility in the supply of labour. As a result, careers tend to become more volatile and they regularly span multiple organizations, positions are more often temporary and people increasingly work on their own account. In this framework, the current job is no longer the most relevant unit to gauge the labour market position of an employee. Rather, the labour market position may be better assessed in terms of the ability to land another job. Security is not found in the current job, but in the ability to find a replacement job if needed. Employability is the key to ensuring such flexicurity, as Wilthagen and Tros (2004) coined the amalgamation of flexible and secure labour markets. The development of transferable skills, among others through non-formal education, is a crucial driver in sustaining personal employability (Froehlich et al., 2014).

Automation processes are associated with the provision of training in two ways. First, both the effects of automation and the provision of training are unequally distributed across the labour market population. Automation negatively affects low-skilled employees primarily (Graetz and Michaels, 2018; Autor, 2019), while this group is also the least likely to receive training (OECD, 2017). In other words, automation exacerbates the urgency to improve the employability for a group that likely does not have the tools to do so. Second, the likelihood of receiving training may be influenced by the probability that the job is automated. Intuitively, the argument is that firms may be less likely to invest in employees that hold jobs that may become redundant in the near future. The next sections explore both effects in more detail and we argue that both effects are influenced by the institutional context in which they take place.

\subsection{The provision of training across skill levels}

The current understanding of non-formal education provision in the labour market heavily leans on the seminal work by Becker (1964). Becker introduced the notion that training and the resulting skills lie on a continuum. On the one extreme, training is generic and the resulting skills are easily transferred, without loss of value, to other work contexts. On the other extreme, training is specific and the resulting skills cannot be transferred to other work contexts. They are only valuable in the current work and training context. In a fully competitive market, workers invest in generic training as it increases their market value. Firms, in contrast, do not provide generic training as it automatically leaks away to other work contexts and they cannot reap the benefits. Specific training increases both the current marginal productivity of the worker and the competitiveness and profits of the firm. Also, by 
IJM

42,8

1400

definition, it cannot leak away to other firms and therefore workers and the firm share the costs for specific training. In this framework, there is no underinvestment in training and all workers will be able to receive the appropriate amount of training.

A market governed by imperfect competition is a more realistic assumption if only because "generic" skills can be applied in a limited number of firms only (Stevens, 1994; Booth and Snower, 1996). In imperfect markets, firms have bargaining power regarding wages and, as a result, wages now imperfectly follow the productivity levels of workers. Also, there are transfer costs between firms and information asymmetry, which makes it problematic for workers to appropriate investments in training. At the same time, firms remain reluctant to invest in generic training, as the benefits may still leak away to other firms. As a result, Becker's predicted outcomes change. Underinvestment in training is more likely (Stevens, 1994) and access to training may depend on the current skill-level of the worker.

The most prominent argument for explaining differences in the provision of training across skill-levels is that budget constraints limit the access to training, particularly for those at the lower end of the wage distribution (Acemoglu, 1996), given a certain level of consumption on other goods and services. This argument is valid mainly in the context of training that is not provided by the firm. However, low-wage workers also have fewer possibilities in sharing training costs with the employer, for example in terms of a temporary wage cut, when employer and employee work together in the provision of training. Acemoglu and Pischke (1999) underline the role of the institutional context in understanding the provision of training across the skill distribution and they argue that the effect is mitigated in imperfect markets with a strong wage compression (Acemoglu and Pischke, 1999). Wage compression is the phenomenon that the wage returns to productivity decline when the skill level of an employee increases: Higher skilled employees earn relatively little compared to their productivity - the wages are compressed - and this incentivizes firms to provide training in the lower end of the skill distribution in order to increase the mean skill level of their employees. Acemoglu and Pischke (1999) then show that institutional differences between countries influence the level of wage compression and consequently the provision of training by firms. In countries with regulated labour markets, wage compression tends to be more pronounced, which incentivizes firms to provide training. Smaller wage compression indicates markets that are more competitive and mimic more closely the outcomes in Becker's original model. Providing training falls upon the workers primarily. Particularly in the case of budget constraints in combination with a faltering credit market, there is the risk of underinvestment in training. A lack of alternative jobs in which the training can be appropriated further discourages investment in training. These effects are most pronounced at the lower end of the wage distribution.

Empirical studies corroborate that the incidence of training increases with skill level and the correlated wage level. Focussing on training provided by the firm, Osterman (1995) shows that US professional/technical workers receive more training than blue collar workers. Regardless the type of workers, training provision increases with the skill level involved in the job. Interestingly, the involvement of unions in the sector increases the likelihood that training is provided. This is consistent with the idea that a more regulated labour market leads to wage compression and consequently incentivizes the provision of training, particularly at the lower end of the skill distribution. The OECD (2017) also documents a persistent gap in training between lower skilled and higher skilled employees. Lower skilled employees have fewer resources to engage in training and the returns to training are deemed relatively low. Bassanini et al. (2005) find the same for European countries and they state that "learning begets learning" (p3). Stressing the importance of the context, they document significant differences across European countries in the incidence of non-formal education. Finally, Campaner et al. (2018) assess the provision of on-the-job training in Germany. They find that flexible working environments require more training and also provide it more often. At the same time, the provision of training is skewed towards higher skilled employees. 
Partially, the provision of training reflects the technological advances made in the organizations and employees working with new technology receive more training. This is evidence of a direct link between technological change in the job, including risk of automation and training provision.

\subsection{Automation and the provision of training}

The introduction of new technologies, with automation as an example, may also have a separate effect on the provision of training. Suppose, a newly introduced technology increases the productivity of high-skill workers relative to low-skill workers. In competitive markets, with limited wage compression, training will be directed to the most productive employees and training of low-skilled employees becomes less attractive. In the case that the new technology makes low-skilled jobs less productive, wages fall and low-skilled workers will be less able to invest to ascertain training. Consequently, employability can fall in this situation. In contrast, firms that operate in a market with more wage compression will have an incentive to invest in training at all skill levels.

Another argument follows directly from the combined complementarities between both skill level and on-the-job training and skill level and technological change (Brunello, 2004). If on-the-job training is provided more at higher skill levels and technological change increases the relative demand for high-skill jobs, the relative demand for training will increase at the higher end of the skill distribution.

Nedelkoska and Quintini (2018) provide an empirical example for OECD countries and they test whether the risk of automation is related to several types of training. They indeed find that after controlling for initial educational attainment, age, ICT use on the job and country dummies, the risk of automation depresses the provision of on-the-job training, as well as that of other types of training.

It is important to note that at the firm level, the causality can also run in the other direction with the organizational human capital strategy, including training, being predictive of innovation. Firms aiming to innovate may invest in the skills towards the innovation, though training may still be provided asymmetrically across the skill distribution. The current skill distribution in a firm may influence the adoption of new technologies (Piva and Vivarelli, 2009; Jiménez-Jiménez and Sanz-Valle, 2005; Bresnahan et al., 2002) and with that also the exposure to new technologies and the need to invest in training for its workers across all skill levels.

\subsection{Expectations}

This leads to a number of expectations regarding the provision of training in Europe. First, the incidence of training is expected to be lowest for low-skilled employees, who also face the highest risk of job loss due to automation. Second, the risk of automation has an independent effect on the incidence of training; the returns to training are low if the risk of automation is high, which prevents both firms and employees to invest in training. Third, these effects depend on the labour market environment. Country-level differences in the labour market structure as well as its institutional set-up may influence these expectations. We expect higher levels of training attendance and smaller difference in training attendance between high- and low-automation risk workers, in countries with a more regulated labour market and more active labour market policies.

\section{Empirical strategy}

\subsection{Intuition}

The analysis aims at identifying the association between the workers' probability to attend training and the automation risk of their main job. For this, occupations are scored on their
Non-formal education and job automation risk

\section{1}


IJM

42,8

automation risk using the measures developed by Frey and Osborne (2017). In the next step, we divide workers into three groups based on their jobs' automation risk, identifying high-, medium- and low-risk workers. We then compare the incidence of training in the three groups by country. Finally, we estimate logistic regression models to explore the relationship between training attendance, automation risk and country-level characteristics net of salient characteristics. The analyses make use of the microdata of the 2016 European Labour Force Survey (EU-LFS).

\subsection{Estimating automation risk}

There is considerable debate about which method is most appropriate for assessing the susceptibility of jobs to automation. Frey and Osborne (2017) assume that each occupational title is described as a combination of eight task groups. Arntz et al. (2016), instead, suggest that the actual tasks performed in an occupation may vary considerably across firms, industries, geography and skill levels. Each occupational title may thus hide a considerable variation in the actual task profile. Assessing the susceptibility to automation is then best done at the level of the task, using information on the actual tasks performed, rather than on an assumed task-profile for each occupation. The two approaches lead to different estimates of the number of jobs at risk to automation. Frey and Osborne (2017) see $47 \%$ of jobs in the USA disappear, while Arntz et al. (2016) predict an average job loss of $9 \%$ across 21 OECD countries. Nedelkoska and Quintini (2018) refine the task-based approach by Arntz et al. (2016) while extending the coverage to 32 OECD countries. They find that, on-average, $14 \%$ of the jobs have a high probability to become redundant through automation.

As the data available for this project have occupational information only, we cannot adopt a task-based approach and we rely on the index developed by Frey and Osborne (2017). Each occupational code in the dataset is scored on its likelihood of automation. Frey and Osborne have developed the index for the 702 occupations in the Standard Occupational Classification (SOC) in the USA. The index derives from an iterative procedure in which experts scored 70 occupations on their susceptibility to automation. Frey and Osborne then use information from the $\mathrm{O}^{*} \mathrm{NET}$ database to describe each occupation as a specific combination of eight predetermined task groups. The combination of task groups is then used as a predictor for the experts' evaluations of the occupation's susceptibility to automation. Frey and Osborne conclude that the model based on the eight task groups is a good predictor of the experts' evaluation, and the model is then extrapolated to cover the entirety of the 702 SOCoccupations. The result is an index between zero and one that indicates increasing susceptibility to automation of each of the 702 SOC-occupations.

In the current study, the index has been translated from the SOC to the 2008 International Standard Classification of Occupations (ISCO-08) available in the EU-LFS using the crosswalks of the US Bureau of Labor Statistics [2]. The 702 SOC-occupations collapse into 1263 -digit ISCO-08 occupations. The automation risk score of the 126 ISCO-08 occupations is the average of the corresponding SOC occupations.

By adopting the index of Frey and Osborne (2017), the analysis implicitly assumes a relatively high - given the alternative methods - average risk-profile for the occupations. In the analysis, however, the absolute risk level is less crucial to the outcome. More important is the relative ordering of the occupations and the labour market behaviour across the distribution of automation risk. To mitigate a potential upward bias, while retaining information on the distribution of occupations on the $0-1$ scale, we collapse occupations into three groups (high-, medium- and low-automation risk). Following Frey and Osborne (2017), we code occupations with an automation score above 0.7 as high-risk. We define occupations with an automation score below 0.3 as low-risk, while the medium group includes the remaining occupations. 


\subsection{Data and sample selection}

We use data from the 2016 EU-Labour Force Survey. The EU-LFS is a harmonized survey conducted yearly in the countries of the European Union and it collects detailed information on individuals' socioeconomic, demographic and employment characteristics. As we rely on three-digits ISCO codes to compute the key explanatory variable, automation risk, we drop 4 of the 31 countries in the original dataset (Bulgaria, Malta, Poland and Slovenia), as they do not provide detailed occupational codes. For the same reason, the analyses only pertain to individuals in paid (self-)employment. With this, the also relevant group of unemployed possibly as a result of automation - and their efforts and human capital investments to improve employability is not included. In addition, Switzerland, Cyprus, Iceland and the United Kingdom have been excluded from the analysis because of missing information on key country-level characteristics.

\subsection{Principal variables}

The analysis aims at estimating the association between automation risk and training attendance. We expect the relationship to be moderated by country-level differences in labour market policies. Given this goal, there are six principal variables of interest (Table 1).

The dependent variable is Non-Formal Education, corresponding to the COURATT variable in the EU-LFS dataset [3], which indicates if someone attended non-formal education or training in the four weeks preceding the interview. As the variable refers to a short time span, mean training attendance is relatively low, compared for example to OECD estimates (OECD, 2017).

The main explanatory variable, Automation risk, is a three-way ordinal variable that indicates whether somebody holds a job that has a relatively high-, medium- or low risk of being automated in the future. As previously discussed, we follow an approach assessing a scenario of maximum exposure to automation (the technological frontier), while the current urgency (governed by organizational processes) may be in fact smaller. The association between automation risk and training is then a conservative estimate.

We use three proxies for the level of regulation in the countries' labour markets. The first two are the Employment Protection Legislation (EPL) for regular employment and for temporary contracts (EPL regular contracts and EPL temporary contracts), as measured by the OECD. The third variable indicates the public spending on Active Labour Market Policies

\begin{tabular}{ll}
\hline Dependent variable & Description \\
\hline Non-formal education & $\begin{array}{l}\text { EU-LFS variable COURATT: "Did you attend any courses, seminars, conferences or } \\
\text { received private lessons or instructions outside the regular education system within } \\
\text { the last } 4 \text { weeks?" }\end{array}$
\end{tabular}

Main independent variables

Job's automation risk This is a variable dividing workers in three automation risk groups: low (less than LMP Spending $30 \%$ ), medium (between 30 and $70 \%$ ) and high (more than $70 \%$ )

EPL, regular

contracts This is the country-level expenditure on active labour market policies, aimed at training, as a percentage of GDP (standardized), source: OECD (2019a) The strictness of Employment Protection Legislation for regular contracts. This is a synthetic variable ranging from $0-6$ with 6 indicating the strictest employment protection legislation, source: OECD (2019b)

EPL, temporary contracts The strictness of Employment Protection Legislation for temporary contracts. This is a synthetic variable ranging from $0-6$ with 6 indicating the strictest employment protection legislation, source: OECD (2019c)

Country
Non-formal education and job automation risk

1403

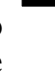


IJM

42,8

1404

related to training as percentage of the country's GDP (LMP Spending), also made available by the OECD. Although significant differences exist in how countries distribute LMP spending and how LMP are implemented, public expenditure on LMP is a proxy of a proactive role of the national government in regulating labour market dynamics.

In addition, to assess country level differences in the effects, we use country dummies to account for unobserved structural circumstances that may influence the incidence of training directly. At the same time, contextual factors may moderate the effect of automation on training followed.

\subsection{Empirical approach}

The empirical analysis explores, in two steps, the association between automation risk in the main job and the probability to attend training. The first step is a descriptive analysis that unveils country level differences in the provision of training to the three risk groups. The second step is a logistic regression that estimates the incidence of training while controlling for characteristics of the employees and country-level characteristics. The regression analysis allows to abstract from composition effects of people that hold jobs that have a high risk of automation. In addition, the direct and mediating effect of contextual factors can be assessed. We adopt a set of individual and country level confounding factors that are associated with training attendance (see Table A1 in the Appendix for the variable summaries).

As the EU-LFS is a repeated cross-sectional survey, it does not allow to analyse how individual-level variations in automation risk over time affect the probability to attend training. We are therefore careful to interpret the results in terms of causal effects. However, given the conceptual frame and the cross-country variation that we can exploit, we maintain that the results are suggestive of causal relationships, particularly vis-à-vis country level variations in the association between risk profile of the job and training attendance. The key contextual variables are quite stable over time and as such a panel approach would not likely improve the assessment of the contextual variables. It would rather pick up repeated crosscountry differences. To account for the multilevel nature of the data, the standard errors in the logit models have been clustered at the country level. In addition, all models have also been executed in a multilevel setting (Appendix, Table A3). The conclusions that can be derived from the logit models and multilevel logit models are the same. For ease of exposition, the logit models are presented in the main text. Although the dataset includes sample weights, the results presented in this article are unweighted. Separate analyses with weights have been run, and the results are consistent with the unweighted ones.

At the individual level, on top of the Automation Risk variable, controls are added for Educational Attainment to correct for the idea that training begets training; more highly educated employees typically have more training opportunities on the job as well (Bassanini et al., 2005). The Economic Sector is included to account for sectoral patterns in training provision also accounting for sector differences in the production function and the salience of automation for production (see, for example, Ortega-Argilés et al., 2011). Also, this may go some way in mitigating the possible effects of firm structure in the features of automation and the possible association with training provision. Controls for Age and Gender are added. Income is important, as it influences the budget constraint that employees may face in obtaining training. Unfortunately, income is not available for all countries. Given the focus on the institutional context, we present the models for the full set of countries but excluding the income variable. The sensitivity of the results to including income in the model - for a smaller set of countries - was checked and the alternative models confirm the salient role of income in training provision. At the same time, the role of the main variables of interest in relation to training provision is hardly affected. The additional models including income are available upon request. 


\section{Results}

\subsection{Non-formal education by automation risk}

Figure 1 shows the share of workers by automation risk that recently attended training. The figure shows a clear, negative association between automation risk and the proportion of workers that recently attended non-formal training. In particular, the group with the highest likelihood to attend non-formal education are the workers in jobs with the lowest risks of automation (below $20 \%$ ), as between 14 and $20 \%$ of them has attended a taught activity in the previous four weeks. At the same time, we document the lowest likelihood to attend taught activities (around 5\%) among those in jobs with an automation risk between 70 and $90 \%$. Quite remarkably, the percentage of workers having attended taught activities is double that (over 10\%) among the workers facing the highest automation risk (around 95\%). The pattern found is a first indication that polarization as a result of automation comprises of more than the asymmetric differentials in the remunerations across the labour market. The gap may be sustained and possibly increased through a lack of human capital investment in the employees that are vulnerable on the labour market negatively influencing their employability. The graph also suggests that the $70 \%$ threshold to distinguish between the high-risk group and the medium-risk and low-risk group is indeed a conservative approach.

Figure 2 presents the share of workers that attended non-formal education by automation risk and by country. The graph documents important country differences in workers' attendance of non-formal education: Romania, Croatia, Greece, Slovakia and Ireland have the lowest rates of non-formal education, with attendance rates ranging between 1 and 3\% among low automation risk workers, and between 0 and $2 \%$ among high automation risk workers. At the other end of the spectrum, Denmark, Sweden, Finland and France have the highest rates of training attendance, with $20-32 \%$ of low automation risk workers having attended some taught activity in the previous four weeks, as well as $12-28 \%$ of high automation risk workers. These differences are suggestive of the relevance of contextual factors, possibly connected to national labour market arrangements. The gap in training attendance between high and low automation risk workers also differs across countries. In each country, employees in high automation risk jobs are less likely to attend training than low automation risk workers. The size of the gap, however, is country-specific. In Denmark, for example, overall provision of training is high and the gap between the two extreme groups is relatively low. This can be indicative of a situation in which there are effective incentives and opportunities, for firms and employees across the board, to provide training. Estonia, in

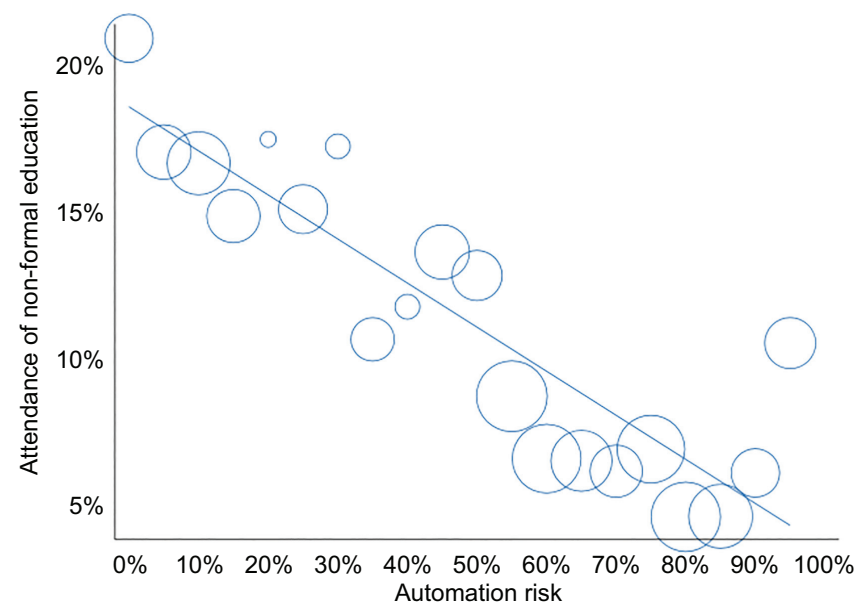

Non-formal education and job automation risk

1405
Figure 1.

Automation risk and training attendance 
IJM

42,8

1406

Figure 2.

Automation risk and training attendance by country

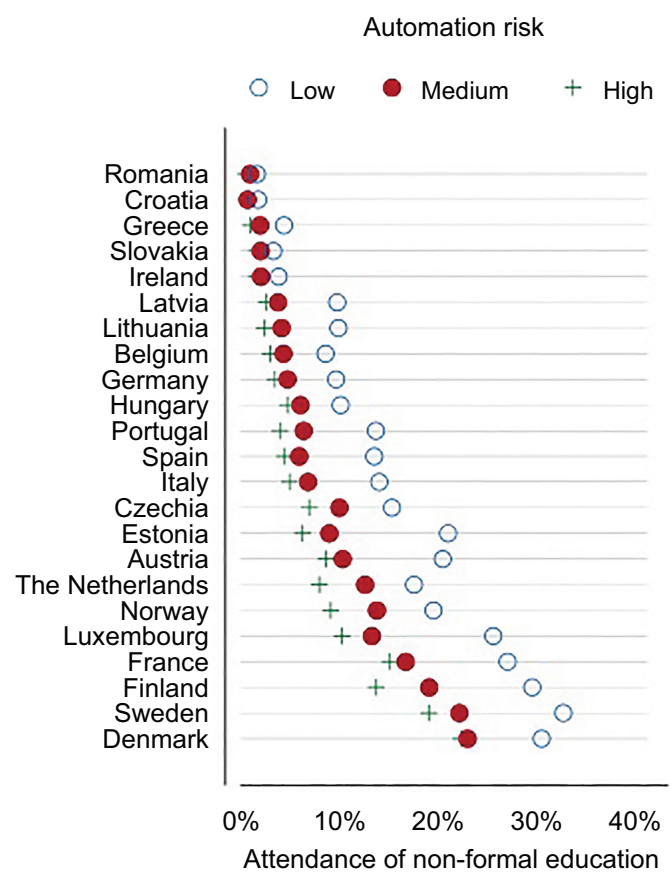

contrast, combines relatively low rates for those in high-risk jobs, with average rates for those in low-risk jobs. In every country, the medium-risk groups sits in between the low-risk and high-risk group in terms of attendance of non-formal training. The medium-risk group tends towards the levels of the high-risk group though which suggests that investments in human capital taper off quickly after a certain threshold of automation risk.

Figure 2 shows a clear variation between countries, but it does not address possible underlying mechanisms that explain the differences between countries. The LMP spending may go some way in explaining the country level differences. Figure 3 shows the scatterplots

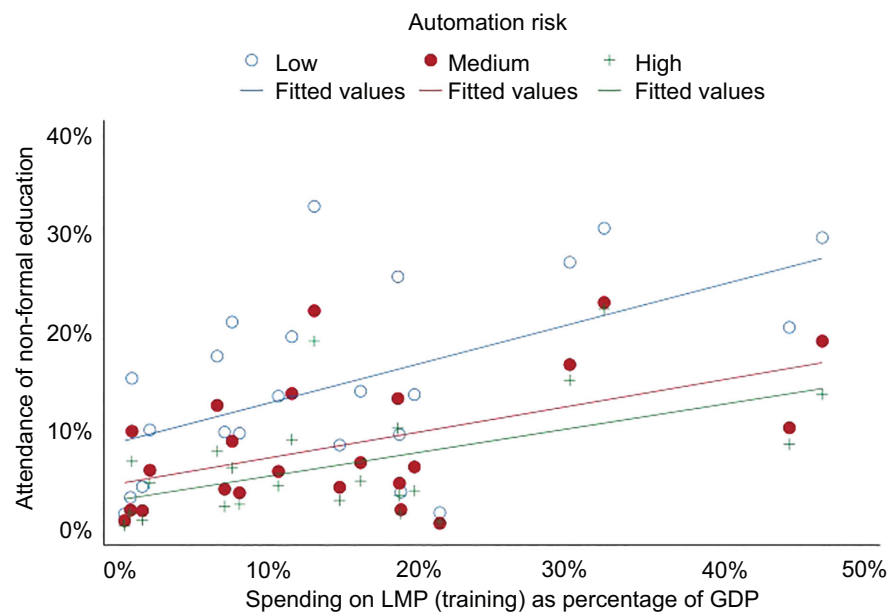

Figure 3.

Automation risk, training attendance and LMP spending 
of LMP spending and the share of attendance of training among the workers in the three groups. There is a clear positive relationship between spending on LMP and the share of attendance of non-formal education for workers in each risk group. The correlation between LMP spending and training attendance is stronger for those in jobs with a lower risk profile, which suggests that LMP spending is directed more often to this group than to the other risk profiles. When excluding the countries with the highest and lowest LMP spending from the analysis, the positive association remains only for the low-risk group (Figure A1 in the Appendix). The patterns found are suggestive of limited effectiveness of labour market policies towards improving life-long learning strategies for those in jobs with medium to high risk of automation.

\subsection{Multivariate analysis}

The bivariate analyses indicate that high automation risk workers are less likely to attend training than workers with lower automation risks. This suggests a sustained polarization on the labour market that unfolds, at least partially, in the context of automation of production and services. If anything, employees at the receiving end of the challenges that come with automation should invest in their human capital in order to increase their employability. In the European context, they do not. This is not to say, though, that automation processes necessarily exacerbate the problematic labour market position of certain groups. There may be a composition effect if certain groups that invest little in training typically hold jobs that have a high probability of being automated. In the logit and multi-level regressions, such composition effects are accounted for.

Table 2 presents the abridged results of eight logistic regression models that explain recent training activity. The full models as well as the multilevel models can be found in the Appendix (Tables A2 and A3). Model 1 in Table 2 presents the predicted effect of automation risk on having attended training in the previous four weeks, controlling only for individual characteristics and country dummies. Models 2 to 4 include, respectively, spending in LMP, EPL for regular employment and EPL for temporary contracts, to account for country level differences in institutional arrangements. Finally, models 5 through 8 assess whether the association between high automation risk and attendance of non-formal education is moderated by the country level variables.

All models confirm that workers in high and medium automation risk jobs have a substantially lower chance of having followed a training in the past four weeks compared to those in a low-risk job. Controlling for individual characteristics and country (Model 1), workers in medium-risk jobs have a $22 \%$ lower odds of attending training than workers in low-risks jobs, and the odds for workers in high-risk jobs are even $31 \%$ lower than for lowrisk workers. This corresponds to an average difference of 2.4 percentage points in the probability of attending training between high- and low-risk workers, and of 1.7 percentage points between medium- and high-risk workers. Given the overall mean of $10 \%$ training attendance (Table A1), the difference between the automation groups is substantive.

Models 2 through 4 underline the importance of country-level characteristics for workers' attendance of non-formal training. The results of model 2 suggest that, as expected, workers are more prone to attend training in countries with a higher LMP spending on training. The level of labour market regulation for temporary contracts (Model 4) also has a positive effect on training attendance, which suggests that training is more likely in a regulated labour market than in a more liberal set-up. This is consistent with the idea that in a free market setting, investments in training are done primarily in a smaller portion of the employees, those for which the costs can be recouped relatively easily.

Apart from the direct effect of countries' labour market institutions on training, they can also play a moderating role in the effect of automation on training attendance. Institutional
Non-formal education and job automation risk

1407 
IJM
42,8

1408

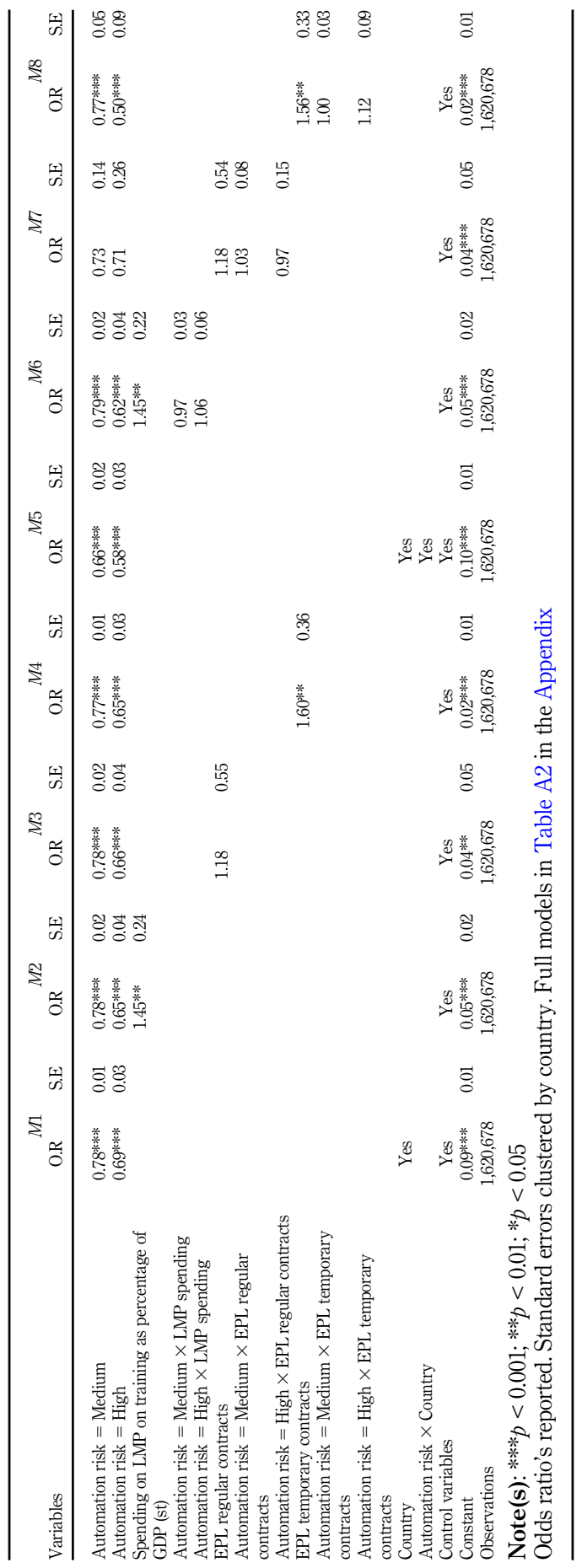

Table 2.

Logistic regressions

explaining non-formal education attendance (abridged) 
arrangements may exacerbate or mitigate the negative association between a high risk of automation and attending non-formal education. This is assessed in models 5 through 8 by adding interaction variables between the country-level variables (Country, LMP, EPL for regular and for temporary contracts) and automation risk. Figure 4 (based on model 5) shows the predicted probability of having attended training in the previous four weeks by automation risk and country, net of other individual characteristics. This model confirms the results of the bivariate analyses in that we observe a significant variation in the general likelihood to attend taught activities and in the gap between low-, medium- and high-risk workers in the attendance of training. In terms of the gap between those in high-risk jobs and low-risk jobs, the figure suggests two regimes. The first regime is found in 6 of the 23 countries included, and in these countries, there is no significant difference in the provision of non-formal education to those in low-risk jobs and those in medium-risk or high-risk jobs. Net of individual characteristics, having a high-risk job does not imply a further penalty in terms of investments in non-formal education. Note, however, that the five countries with the lowest attendance rates in non-formal training are all in this group suggesting that investment in human capital is low allaround. The second regime is found in 13 countries and in these countries, there is indeed a "non-formal education penalty" for those in jobs with a high or medium risk of being affected by automation. Finally, Denmark stands out on its own, as it is the only country in which, net of individual characteristics, workers in high and medium-risk jobs are significantly more likely to have attended training compared to low-automation risk workers.

With the exception of Denmark, the results presented in Figure 4 are robust to relaxing the assumption that the control variables have the same relationship with automation risk and training attendance across all countries: The results when repeating the analysis for all countries separately (Appendix, Figure A2) are very similar. In this model specification, Denmark follows the same pattern as countries in the "second regime", with overall high levels of training attendance and low-risk workers being more likely to attend training than workers in jobs at higher risks of automation. Also, throughout the sample, the differences between the three groups seem to be somewhat smaller, if following the same pattern. This indicates that the control variables, such as age, gender and educational attainment interact in a country-specific manner with the relationship between automation and training.

The country dummies capture structural differences in the way that non-formal education is distributed across the labour market. They are, however, not suggestive of any

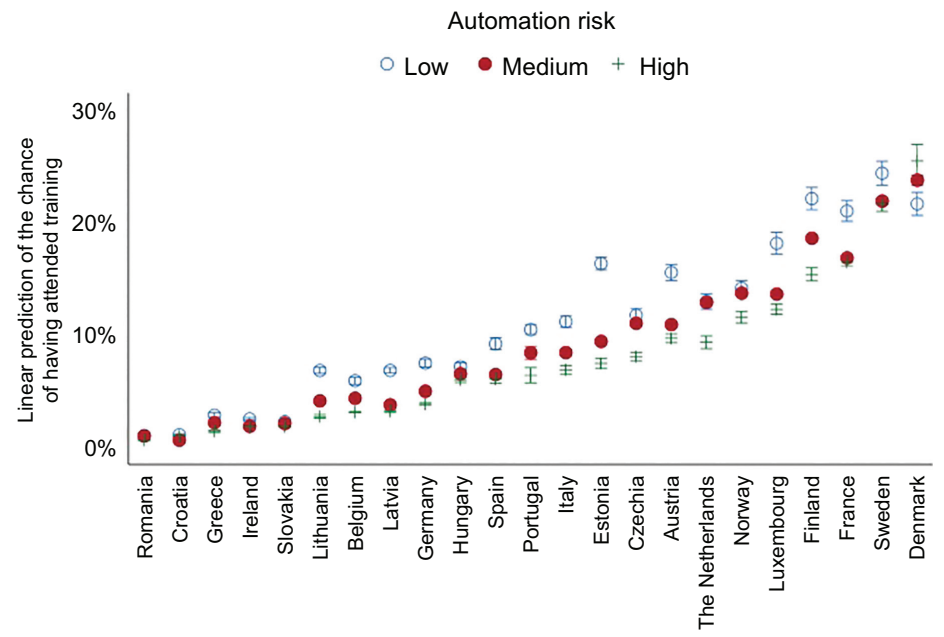

Figure 4 .

Predicted effects by country of automation risk on training attendance 
IJM

42,8

1410

mechanisms that underlie the differences found. The LMP and EPL variables proxy for an active labour market policy and can provide a more substantive interpretation to country differences in the gap of the availability and provision of non-formal education. Models 6 to 8 explore this aspect. We do not find any significant effects of the interactions between automation risk and LMP spending or EPL. This suggests that active labour market policies have a direct association with the overall provision of non-formal training, but not with the distribution of training attendance across jobs with different automation risk profiles.

\section{Conclusion and discussion}

This study has addressed investments, by means of following trainings, in the careers of workers in jobs affected by automation and in particular the country-level differences in this association. With this, the analysis complements studies that assess macro-changes in the job composition, following automation of production by assessing one of the underlying mechanisms. Empirically, investment in the career is specified to having followed a training, other than formal education, in the last four weeks. From the analysis, three main conclusions can be derived.

Workers in jobs that have a relatively high probability of being affected by automation are less likely to have followed non-formal education recently. This is after correcting for composition effects including age, educational level and sector. This study then, using a different empirical approach, different data and considering a different set of countries, extends the empirical evidence for this relationship, which was first established in Nedelkoska and Quintini (2018). The lower training incidence pertains both to jobs with a medium automation risk profile and to jobs with a high risk profile. Firms may be discouraged to invest in workers that will likely be changed or discontinued in the near future, resulting in on-average less training in these groups. Also, there is evidence that workers in automation-prone jobs do not acknowledge the changes that the job may undergo (Coupe, 2019), which suppresses the incentive to invest in the career. Although the career progression of the employees is not explicitly addressed, the result is indicative of a decreased employability of a group in an already vulnerable labour market position.

There are substantial country differences both in the level of attendance of non-formal education, and in the difference in attendance between workers in jobs with a high risk of automation and those faced with a lower risk. Consistently, throughout the 23 European countries in the study, workers in high-risk jobs receive less training than those in low-risk jobs. The incidence of training in the European countries included ranges from $25 \%$ of all workers in Sweden and Denmark to just over 1\% in Croatia, Greece and Romania. The difference between the high- and low-risk group is biggest in Estonia with a $10 \%$ point lower incidence of training in the group in jobs with a high risk of automation. Once controlling for compositional effects, the picture is more diverse. There is a group of countries, with overall low levels of training, registering no additional training penalty for those in jobs that have a relatively high risk of being automated. The second group of countries is characterised by higher levels of training attendance for all workers, but more unevenly distributed between workers with low-, medium- and high-automation risk: in these countries, automation comes with a training penalty for those holding the highest automation risk jobs.

The country differences may be partially explained by active labour market policies that some countries apply. We find a consistent and positive effect of labour market policy spending on the overall attendance of non-formal education. There is no indication, however, that labour market spending influences the distributions of non-formal education across jobs with different automation risk profiles. The policy implications of the analysis are therefore somewhat diffuse. On the one hand, we see clear signs that a pro-active labour market policy is advantageous in boosting a life-long-learning culture compared to the situation in which 
training is left more to the market. The analysis suggests a pro-active role of governments in this respect. On the other hand, the analysis remains silent on the actual arrangements of the policies and their quality. There may thus be hidden heterogeneity in the policy approaches, for example when it comes to targeting specific groups including those affected by automation. Unveiling this heterogeneity may go some way in further detailing which arrangements are more effective than others.

The analysis is suggestive of how the employability of employees in jobs with a high risk of automation develops. To complement the picture, it would be important to assess additional elements of employability including more information on the nature of the training followed, its purpose and also the role of formal education which is particularly salient in the start of the career. Also, following the actual career paths of employees in high-risk jobs in relation to their peers in other jobs would be a valuable next step in assessing their revealed employability. Such a career or panel approach would allow for a stronger empirical test of the causality between potential automation of the job and investments in training. Finally, organizational strategies are important in assessing whether the technical potential of automation - which is measured in automation indices - indeed translates into the factual change in the job arrangements in an organization. Clearly, this has important implications both for the actual perceived risk of automation and the reaction of the employees affected. Confronting an organizational approach to automation as a form of innovation (see, for example, Piva and Vivarelli, 2009; Jiménez-Jiménez and Sanz-Valle, 2005) with the implications it may have for the employees thus seems a fruitful avenue to refine the analysis.

In summary then, the analysis in this paper suggests that labour market polarization extends beyond asymmetric wage dynamics resulting from changed supply and demand for jobs in the wake of automation processes. Polarization also presents itself in investments in careers depending on the likelihood of automation in the current job. This is a worrying sign, as it suggests that employees in jobs affected by automation may become structurally disenfranchised from a changing labour market; their employability decreases. The country differences in training levels are indicative of a spatial polarization in Europe as well. Certain countries, such as the Scandinavian countries, invest in their labour force affected by automation while other countries invest significantly less.

\section{Notes}

1. Non-Formal Education and Training are used interchangeably throughout the text.

2. https://www.bls.gov/soc/ISCO_SOC_Crosswalk.xls

3. Entering formal education may also be a way to shield oneself from the challenges posed by automation. An exploration of the data, however, reveals that it is much less common than nonformal training and that its incidence is heavily skewed towards young employees (15-25). This suggests that the job is a side-activity next to education rather than that education is a response to challenges in the job. That having said, a logistic regression on formal education does document an association, though weaker, with the automation risk in the job similar to that with non-formal education. The model results are available upon request.

\section{References}

Acemoglu, D. (1996), "Credit constraints, investment externalities and growth", in Booth, A.L. and Snower, D.J. (Eds), Aquiring Skills. Market Failures, Their Symptoms and Policy Responses, Center for Economic Policy Research, Cambridge University Press, Cambridge.

Acemoglu, D. and Autor, D. (2011), "Skills, tasks and technologies: implications for employment and earnings", in Ashenfelter, O. and Card, D. (Eds), Handbook of Labor Economics, Elsevier, Amsterdam. 
IJM

42,8

Acemoglu, D. and Pascual, R. (2019), "Automation and new tasks: how technology displaces and reinstates labor", The Journal of Economic Perspectives, Vol. 33 No. 2, pp. 3-30.

Acemoglu, D. and Pischke, J.S. (1999), "Beyond Becker: training in imperfect labour markets", The Economic Journal, Vol. 109 No. 453, pp. 112-142.

Acemoglu, D. and Restrepo, P. (2018), "The race between man and machine: implications of technology for growth, factor shares, and employment", The American Economic Review, Vol. 108 No. 6, pp. 1488-1542.

Arntz, M., Gregory, T. and Zierahn, U. (2016), "The risk of automation for jobs in OECD Countries: a comparative analysis", OECD Social, Employment and Migration Working Papers. No. 189, OECD Publishing, Paris.

Arntz, M., Gregory, T. and Zierahn, U. (2017), "Revisiting the risk of automation”, Economics Letters, Vol. 159, pp. 157-160.

Autor, D.H. (2019), "Work of the past, work of the future. Richard T. Ely lecture", American Economic Association: Papers and Proceeding, Vol. 109 No. 5, pp. 1-32.

Bassanini, A., Booth, A., Brunello, G., De Paola, M. and Leuven, E. (2005), Workplace training in Europe, IZA DP, 1640, Bonn.

Becker, G.S. (1964), Human Capital Theory, Columbia, New York.

Booth, A.L. and Snower, D.J. (Eds) (1996), Acquiring Skills: Market Failures, Their Symptoms and Policy Responses, Cambridge University Press.

Bresnahan, T.F., Brynjolfsson, E. and Hitt, L.M. (2002), "Information technology, workplace organization, and the demand for skilled labor: firm-level evidence", Quarterly Journal of Economics, Vol. 117 No. 1, pp. 339-376.

Brunello, G. (2004), "Labour market institutions and the complementarity between education and training in Europe", in Checchi, D. and Lucifora, C. (Eds), Education, Training and Labour Market Outcomes in Europe, Palgrave Macmillan, London.

Brynjolfsson, E. and McAfee, A. (2014), The Second Machine Age: Work, Progress, and Prosperity in a Time of Brilliant Technologies, WW Norton \& Company, New York.

Calvino, F. and Virgillito, M.E. (2018), "The innovation-employment nexus: a critical survey of theory and empirics", Journal of Economic Surveys, Vol. 32 No. 1, pp. 83-117.

Campaner, A., Heywood, J.S. and Jirjahn, U. (2018), "Flexible work organization and employer provided training: evidence from german linked employer-employee data”, GLO Discussion Paper, No. 233, Global Labor Organization (GLO), Maastricht.

Capone, G., Malerba, F., Nelson, R.R., Orsenigo, L. and Winter, S.G. (2019), "History friendly models: retrospective and future perspectives”, Eurasian Business Review, Vol. 9 No. 1, pp. 1-23.

Cohen, W.M. and Levinthal, D.A. (1990), "Absorptive capacity: a new perspective on learning and innovation”, Administrative Science Quarterly, Vol. 35 No. 1, pp. 128-152.

Coupe, T. (2019), "Automation, job characteristics and job insecurity", International Journal of Manpower, Vol. 40 No. 7, pp. 1288-1304.

Dosi, G. and Mohnen, P. (2019), "Innovation and employment: an introduction", Industrial and Corporate Change, Vol. 28 No. 1, pp. 45-49.

Frey, C.B. and Osborne, M.A. (2017), "The future of employment: how susceptible are jobs to computerisation?", Technological Forecasting and Social Change, Vol. 114, pp. 254-280.

Froehlich, E.D., Beausaert, S., Segers, M. and Gerken, M. (2014), "Learning to stay employable", Career Development International, Vol. 19 No. 5, pp. 508-525.

Fugate, M., Kinicki, A.J. and Ashforth, B.E. (2004), "Employability: a psycho-social construct, its dimensions, and applications", Journal of Vocational Behavior, Vol. 65 No. 1, pp. 14-38.

Goos, M. and Manning, A. (2007), "Lousy and lovely jobs: the rising polarization of work in Britain", The Review of Economics and Statistics, Vol. 89 No. 1, pp. 118-133. 
Graetz, G. and Michaels, G. (2018), "Robots at work", The Review of Economics and Statistics, Vol. 100 No. 5 , pp. $753-768$.

Hou, J., Huang, C., Licht, G., Mairesse, J., Mohnen, P., Mulkay, B., Peters, B., Wu, Y., Zhao, Y. and Zhen, F. (2019), "Does innovation stimulate employment? Evidence from China, France, Germany, and The Netherlands", Industrial and Corporate Change, Vol. 28 No. 1, pp. 109-121.

Jiménez-Jiménez, D. and Sanz-Valle, R. (2005), "Innovation and human resource management fit: an empirical study", International Journal of Manpower, Vol. 26, pp. 364-381.

Kerr, S., Maczulskij, T. and Maliranta, M. (2020), "Within and between firm trends in job polarization: the roles of globalization and technology", Journal of Economic Geography, Vol. 20 No. 4, pp. 1003-1039.

Malerba, F., Nelson, R.R., Orsenigo, L. and Winter, S.G. (2016), Innovation and the Evolution of Industries: History Friendly Models, Cambridge University Press, Cambridge.

Nedelkoska, L. and Quintini, G. (2018), "Automation, skills use and training”, OECD Social, Employment and Migration Working Papers. No. 202, OECD Publishing, Paris.

OECD (2017), OECD Employment Outlook 2017, OECD Publishing, Paris.

OECD (2019a), Labour Market Programmes: Expenditure and Participants, OECD Employment and Labour Market Statistics (Database), OECD Publishing, Paris, doi: 10.1787/data-00312-en.

OECD (2019b), Employment Protection Legislation: Strictness of Employment Protection Legislation: Regular Employment, OECD Employment and Labour Market Statistics (Database), OECD Publishing, Paris, doi: 10.1787/data-00318-en.

OECD (2019c), Employment Protection Legislation: Strictness of Employment Protection Legislation: Temporary Employment, OECD Employment and Labour Market Statistics (Database), OECD Publishing, Paris, doi: 10.1787/data-00319-en.

Ortega-Argilés, R., Potters, L. and Vivarelli, M. (2011), "R\&D and productivity: testing sectoral peculiarities using micro data”, Empirical Economics, Vol. 41 No. 3, pp. 817-839.

Osterman, P. (1995), "Skill, training, and work organization in American establishments", Industrial relations: A Journal of Economy and Society, Vol. 34 No. 2, pp. 125-146.

Pellegrino, G., Piva, M. and Vivarelli, M. (2019), "Beyond R\&D: the role of embodied technological change in affecting employment", Journal of Evolutionary Economics, Vol. 29 No. 4, pp. 1151-1171.

Piva, M. and Vivarelli, M. (2009), “The role of skills as a major driver of corporate R\&D”, International Journal of Manpower, Vol. 30, pp. 835-852.

Stevens, M. (1994), “A theoretical model of on-the-job training with imperfect competition”, Oxford Economic Papers, Vol. 46, pp. 537-562.

Wilthagen, T. and Tros, F. (2004), "The concept of 'flexicurity': a new approach to regulating employment and labour markets", Transfer: European Review of labour and research, Vol. 10 No. 2, pp. 166-186.

Wittekind, A., Raeder, S. and Grote, G. (2010), "A longitudinal study of determinants of perceived employability”, Journal of Organizational Behavior, Vol. 31 No. 4, pp. 566-586. 
$\mathrm{IJM}$
42,8

Appendix

\begin{tabular}{llllll}
\hline Variable & $N$ & Mean & SD & Min & Max \\
\hline
\end{tabular}

Attendance of non-formal education

$1,620,678 \quad 0.10 \quad 0.30$

1414

Automation risk

Low

$\begin{array}{lll}1,620,678 & 0.25 & 0.44\end{array}$

Medium

$\begin{array}{lll}1,620,678 & 0.42 & 0.49\end{array}$

High

Female

$1,620,678 \quad 0.33 \quad 0.47$

Age

15 to 24

$\begin{array}{lll}1,620,678 & 0.47 & 0.50\end{array}$

25 to 34

35 to 49

50 to 64

$65+$

$\begin{array}{lll}1,620,678 & 0.08 & 0.27\end{array}$

$\begin{array}{lll}1,620,678 & 0.18 & 0.39\end{array}$

$\begin{array}{lll}1,620,678 & 0.39 & 0.49\end{array}$

$\begin{array}{lll}1,620,678 & 0.32 & 0.47\end{array}$

Educational level

Lower secondary

Upper secondary

Tertiary

$1,620,678 \quad 0.03 \quad 0.16$

Economic sector

Agriculture

Manufacturing

Construction

Retail

Logistics

Hospitality

Business services

$\begin{array}{lll}1,620,678 & 0.20 & 0.40\end{array}$

$1,620,678 \quad 0.49 \quad 0.50$

$1,620,678 \quad 0.31 \quad 0.46$

Public services

Other services

Spending on LMP on training as percentage of GDP

$\begin{array}{lll}1,620,678 & 0.06 & 0.24\end{array}$

$\begin{array}{lll}1,620,678 & 0.17 & 0.38\end{array}$

$\begin{array}{lll}1,620,678 & 0.07 & 0.25\end{array}$

$\begin{array}{lll}1,620,678 & 0.14 & 0.34\end{array}$

$1,620,678 \quad 0.05 \quad 0.22$

$\begin{array}{lll}1,620,678 & 0.05 & 0.21\end{array}$

$\begin{array}{lll}1,620,678 & 0.15 & 0.36\end{array}$

$\begin{array}{lll}1,620,678 & 0.27 & 0.44\end{array}$

(standardised)

Table A1.

EPL regular contracts

$1,620,678$

$1,620,678$

$0.05 \quad 0.22$

$\begin{array}{llll}0.29 & 0.91 & -1.00 & 2.64\end{array}$

Summary statistics

EPL temporary contracts

$\begin{array}{lllll}1,620,678 & 2.45 & 0.36 & 1.77 & 3.03\end{array}$

$\begin{array}{lllll}1,620,678 & 2.20 & 0.61 & 0.54 & 3.83\end{array}$




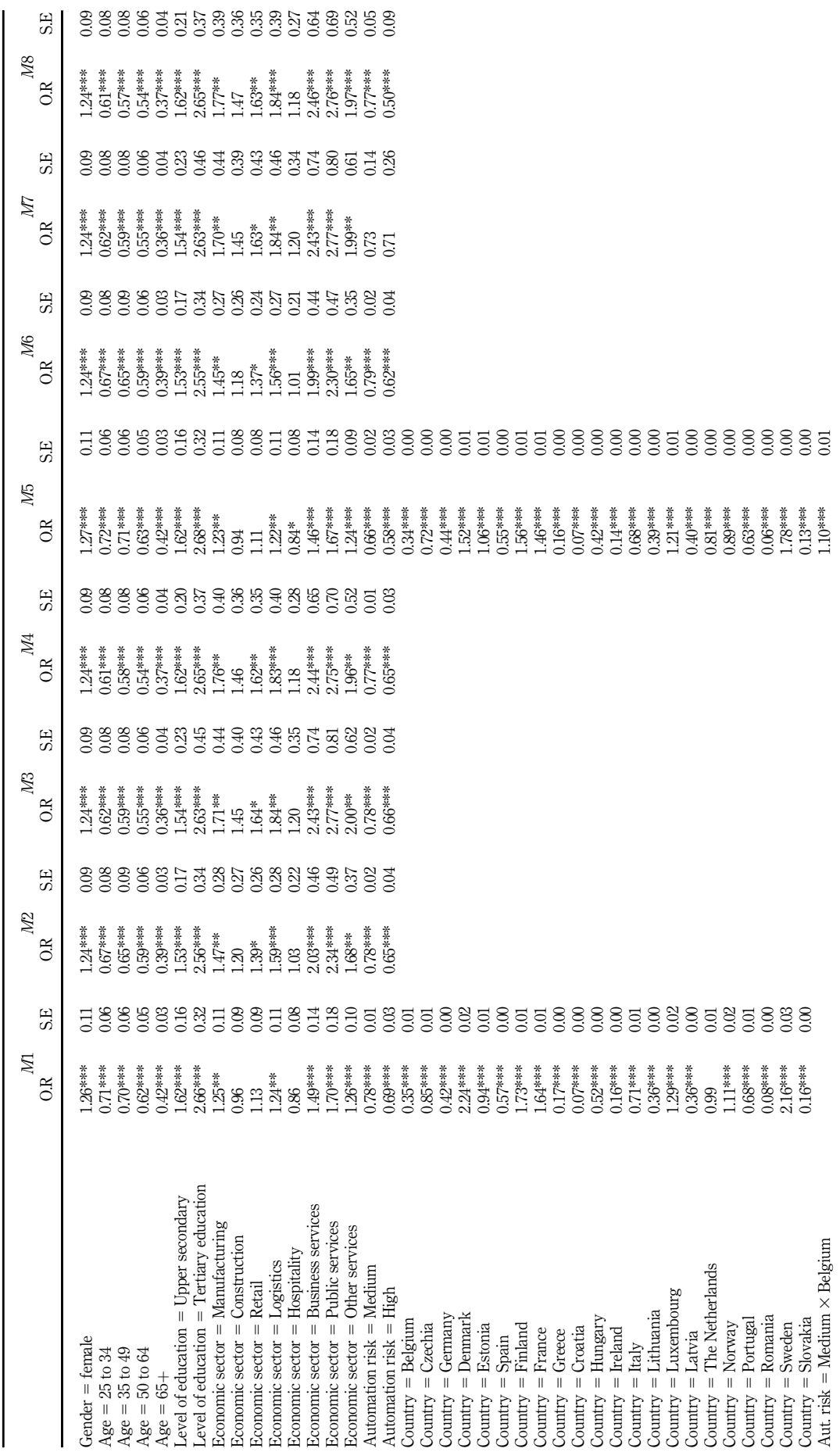

Non-formal
education and
job automation
risk

Table A2. Logistic regressions explaining non-formal education attendance (full model results) 


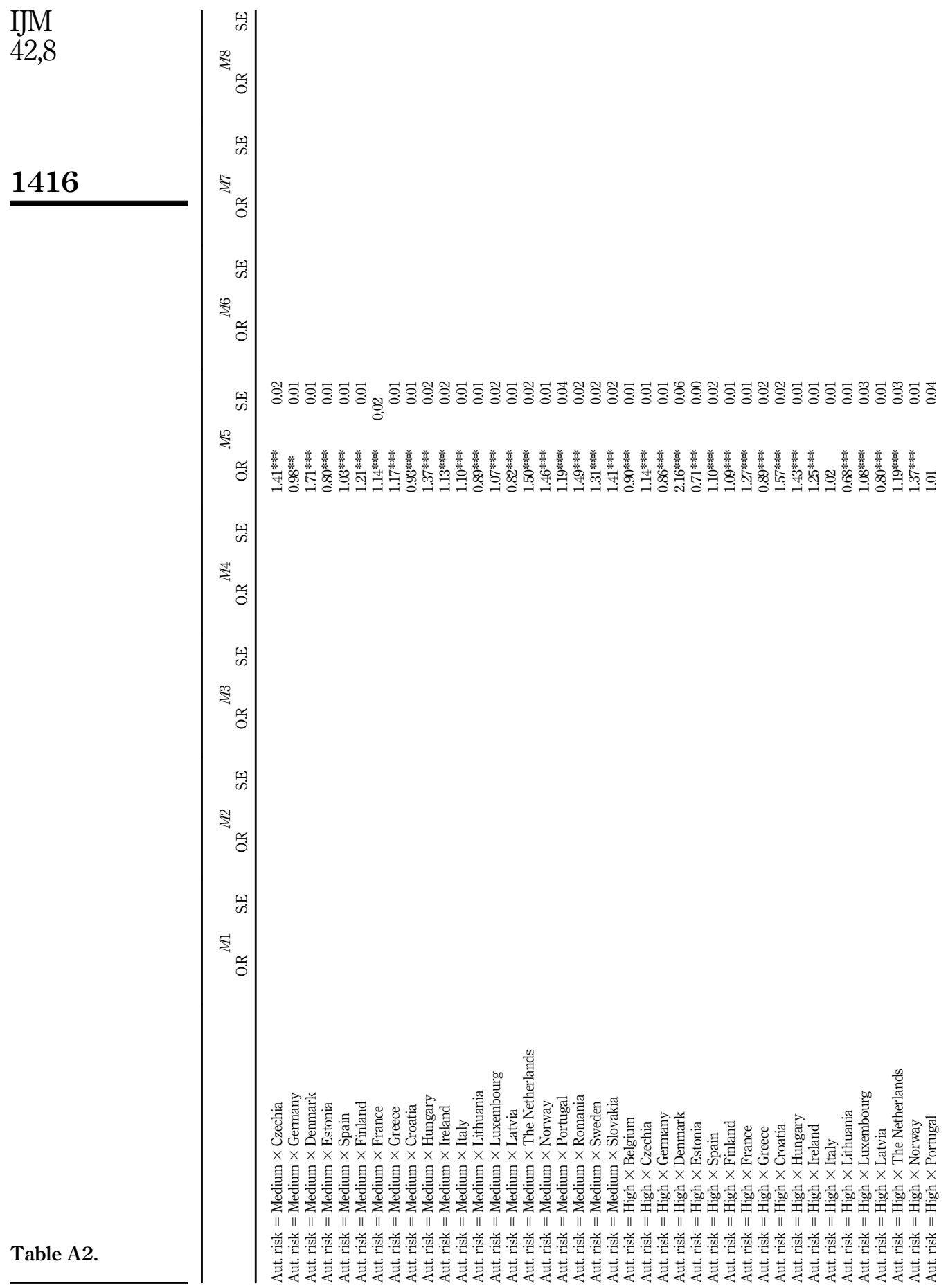




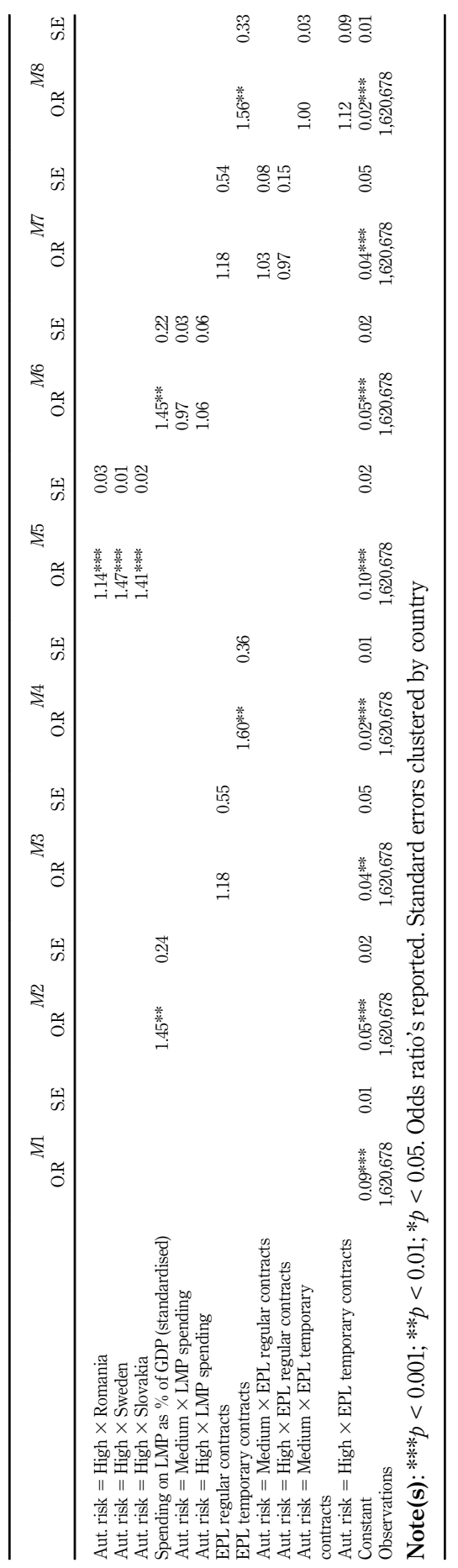

Non-formal education and job automation risk

1417

Table A2. 
IJM
42,8

\section{8}

Table A3.

Multilevel logistic regressions explaining non-formal education attendance

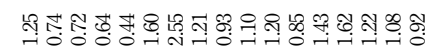

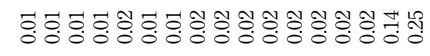

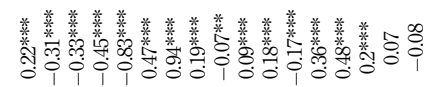

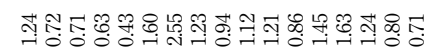

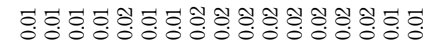

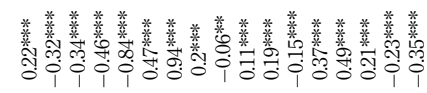

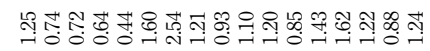

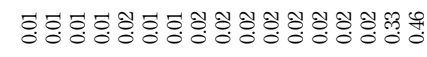

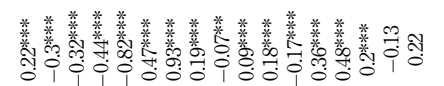

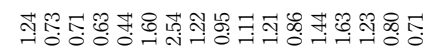

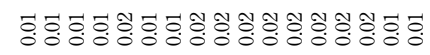

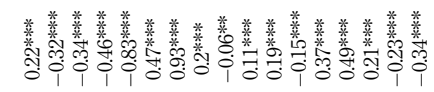

죽영옹

ㄱํㅇ 꿍 ?ํํㅇ

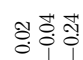

\&.

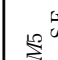

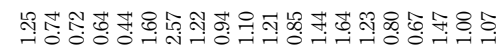

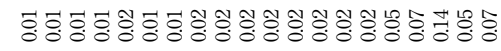

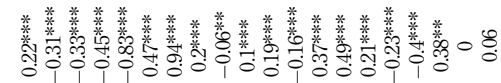

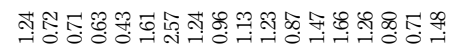

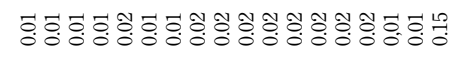

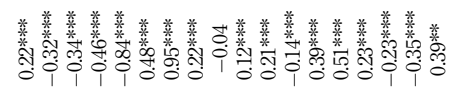

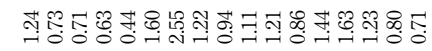

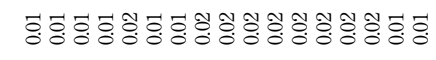

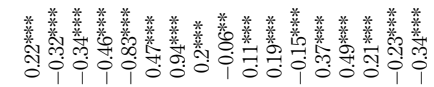

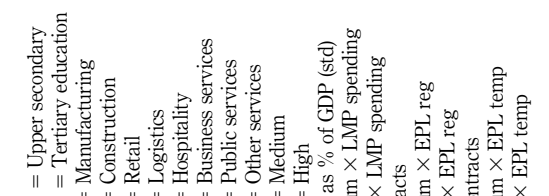

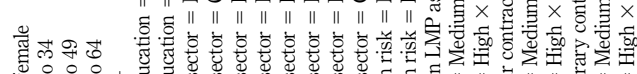

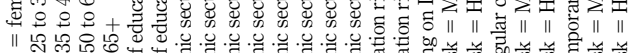

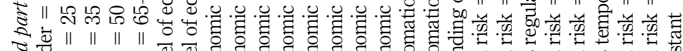

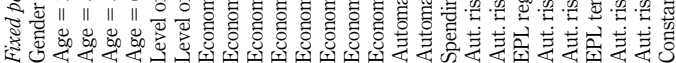

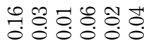

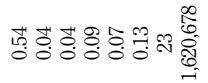

$\stackrel{\infty}{\circ}$

ஜ.

ฉ:

융형융융융융

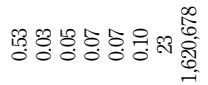

동

:

赵

8

䒿

䔽

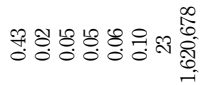

풍

fㅇํ

๙

\&.

है:

䖭

8

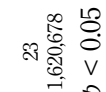

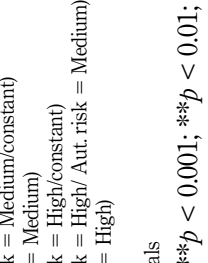

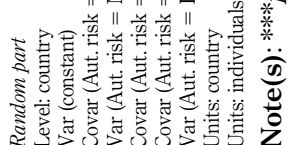




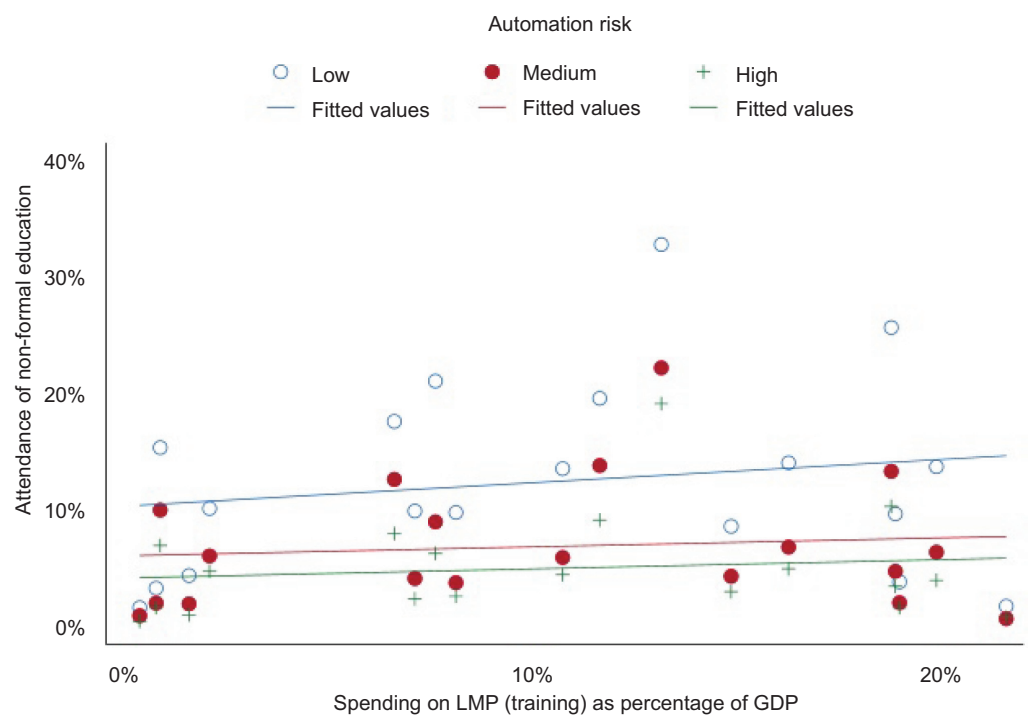

Non-formal education and job automation risk

1419

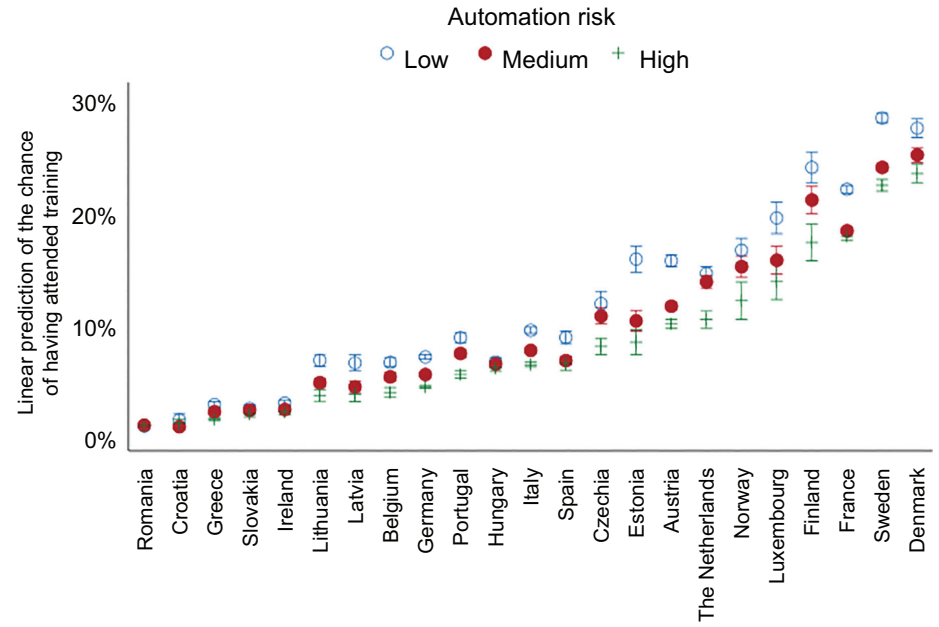

Figure A1.

Automation risk, training attendance and LMP spending (no outliers)

Figure A2. Predicted effects by country of automation risk on training attendance (separate country regressions)

\section{Corresponding author}

Sierdjan Koster can be contacted at: sierdjan.koster@rug.nl

For instructions on how to order reprints of this article, please visit our website:

www.emeraldgrouppublishing.com/licensing/reprints.htm

Or contact us for further details: permissions@emeraldinsight.com 